\title{
Compositional Magnetic Resonance Imaging Measures of Cartilage - Endpoints for Clinical Trials of Disease-modifying Osteoarthritis Drugs?
}

Because compositional magnetic resonance imaging (MRI) techniques enable detection of biochemical and microstructural changes in the cartilage extracellular matrix before gross morphological changes occur, they may be useful as outcome measures for clinical trials focusing on early and potentially reversible disease stages ${ }^{1}$. To date, many of these compositional magnetic resonance imaging (MRI) techniques have not been thoroughly validated in human patients with osteoarthritis (OA) and thus are not presently in routine clinical use. Therefore compositional MRI techniques have rarely been applied in clinical trials ${ }^{2}$, but they have been used with increasing frequency in OA research for "premorphologic" evaluation of cartilage. Techniques comprise relaxometry measurements (T2, T2* and T1rho mapping) including $\mathrm{T} 2 *$ mapping with ultrashort echo-time imaging, sodium imaging, delayed gadolinium-enhanced MRI of cartilage (dGEMRIC), magnetization transfer contrast and glycosaminoglycan (GAG)-specific chemical exchange saturation transfer (gagCEST), diffusion-weighted imaging, and diffusion tensor imaging. Moreover, they seem to have the potential to serve as quantitative, reproducible, noninvasive, and objective endpoints for OA research, particularly in early and preradiographic stages of the disease. Table 1 summarizes available compositional MRI techniques in the context of OA research. Below we describe recent evidence regarding their potential utility.

Numerous clinical studies using T2 mapping have shown that subjects with knee pain have elevated T2 values ${ }^{3}$. Other studies have also associated T2 values with risk factors for OA including age, sex, obesity, and physical activity levels ${ }^{4}$. One study suggested that addition of a T2 mapping sequence to a routine MRI protocol at $3.0 \mathrm{~T}$ improved sensitivity in the detection of cartilage lesions in the knee joint, with only a slight reduction in specificity ${ }^{5}$. A more recent study showed that higher $\mathrm{T} 2$ values at baseline predicted disease onset in a cohort of subjects at risk for $\mathrm{OA}^{6}$.

Clinical studies using T2* mapping to study OA are scarce and offer conflicting results, despite the advantages inherent in 3-D acquisition of T2* sequences. One study of symptomatic and asymptomatic subjects with cavovarus malalignment of the ankle found that $\mathrm{T} 2 *$ values were higher in symptomatic patients than in asymptomatic volunteers ${ }^{7}$. In contrast, in patients with endstage knee OA who had total knee arthroplasty, T2* values of knee articular cartilage decreased with increasing histologic cartilage degeneration ${ }^{8}$. Thus, the utility of $\mathrm{T}^{*} *$ mapping remains to be determined.

It has been suggested that T1rho imaging may be more sensitive than T2 mapping for differentiating between normal cartilage and early stage $\mathrm{OA}^{9}$. A recent study compared parallel changes of quantitative T2, T1rho, and dGEMRIC mapping of human cartilage; T1rho and dGEMRIC mapping seemed to be more sensitive to early stages of cartilage degeneration than quantitative $\mathrm{T} 22^{10}$.

Another technique, dGEMRIC, is sensitive to cartilage proteoglycan GAG content and may predict the development of $\mathrm{OA}^{11}$. It was recently demonstrated that dGEMRIC indices in medial tibiofemoral compartments decrease as the radiographic Kellgren-Lawrence grade increases ${ }^{12}$. Six weeks of immobilization has been shown to result in biochemical changes in the cartilage that are measureable by dGEMRIC. A longitudinal study showed that lower baseline T1Gd values using dGEMRIC in medial and lateral femoral cartilage were associated with a higher grade of joint space narrowing after 11 years, and also with development of osteophytes ${ }^{13}$. Another study found high-grade medial meniscal damage to be associated with lower dGEMRIC indices in the medial tibiofemoral compartment ${ }^{14}$. Longitudinal changes reported in dGEMRIC indices over a 1-year period and their relationship with changes in cartilage thickness in the tibiofemoral compartments evaluated after 2 years demonstrated that a decrease in dGEMRIC indices over 1 year was associated with increased cartilage thickness after 2 years (Figure 1$)^{15}$. Because the majority of the sample in that study had no radiographic OA or mild radiographic $\mathrm{OA}$, the decrease in dGEMRIC (i.e., decrease in GAG) longitudinally was thought to be associated with cartilage swelling, representing initial degeneration of cartilage. However, to date, there has been no strong evidence that changes in dGEMRIC over time predict the incidence or progression of cartilage loss. Of note, Figure 1 illustrates that concurrent degeneration of articular cartilage and meniscus is demonstrable by dGEMRIC, consistent with a recent report ${ }^{16}$.

Clinical studies using sodium MRI are limited. A feasibility study found a higher mean fixed-charge density in healthy individuals compared to symptomatic early OA subjects, in whom proteoglycan loss was indicated ${ }^{17}$. One study ${ }^{18}$ reported that the sodium concentration in both

Personal non-commercial use only. The Journal of Rheumatology Copyright @ 2016 . All rights reserved. 
Table 1. Summary of compositional MRI techniques.

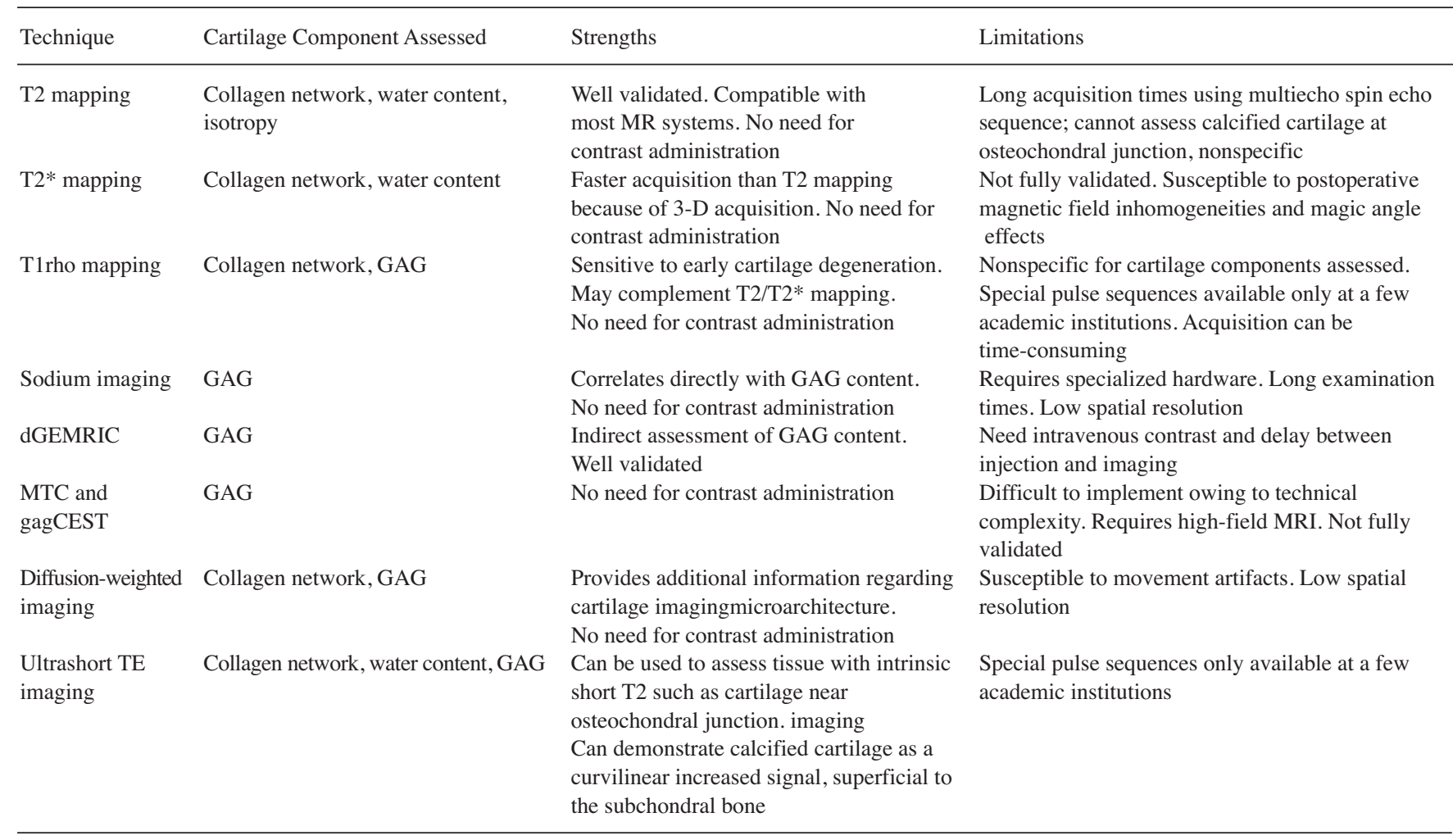

MRI: magnetic resonance imaging; GAG: glycosaminoglycans; dGEMRIC: delayed gadolinium-enhanced MRI of cartilage; gagCEST: GAG chemical exchange saturation transfer; MTC: magnetization transfer contrast; TE: echo time.

healthy and OA knees at $7 \mathrm{~T}$ imaging with fluid-suppressed sodium MRI can be used for detection of OA with good sensitivity and good specificity. Although diffusion imaging may supplement other quantitative techniques for evaluating cartilage, clinical studies that use the technique are rare. However, in 1 study, diffusion tensor imaging showed excellent reproducibility and may be able to differentiate healthy from OA subjects ${ }^{19}$. Clinical studies examining the potential role of gagCEST in the assessment of OA-related cartilage degeneration are lacking. Feasibility studies using in vivo gagCEST have shown gagCEST MRI to be sensitive to GAG levels in the cartilage ${ }^{20}$. The value of this technique in assessment of OA-related cartilage degeneration needs further study.

Some methodological issues regarding application of compositional MRI techniques in OA studies need to be considered. Biomechanical loading or physical stress applied to cartilage may alter measurements of the quantitative composition of cartilage in some techniques, such as T2 mapping and dGEMRIC. Thus, the physical activities of subjects before undergoing compositional MRI may have an effect on the results; however, to date, there is no consensus on how subjects should be prepared. Also, there is no consensus on the best way to segment a defined region of interest within the articular cartilage for compositional analysis. Ideally, all articular cartilage of a defined anatomical structure (e.g., medial femur, tibia, etc.) should be segmented before values of quantitative compositional variables are extracted, which would then reflect the compositional status of the whole region. The use of one or only a few images displaying the articular cartilage from a region or joint of interest will not accurately reflect the compositional status of cartilage for a given region or joint. Further, to assess the predictive effect of cartilage composition in regard to longitudinal structural deterioration, a region-based analysis would be better than a whole joint-based analysis. For example, one would not expect a decrease in the GAG content of the cartilage matrix in the anterior region of the medial tibial plateau to cause cartilage loss in the posterior region of the lateral femoral condyle. This point is of the utmost importance for future studies using compositional MRI techniques. Finally, before the compositional status of cartilage can be put to use as a biomarker for clinical trials involving disease-modifying OA drugs, the predictive effect of longitudinal compositional changes on the incidence or progression of structural deterioration - not just their effect on baseline values or indices - will need to be shown. There has been no strong evidence of such a relationship.

There are limitations of compositional MRI techniques that need to be overcome before they can be applied in larger 


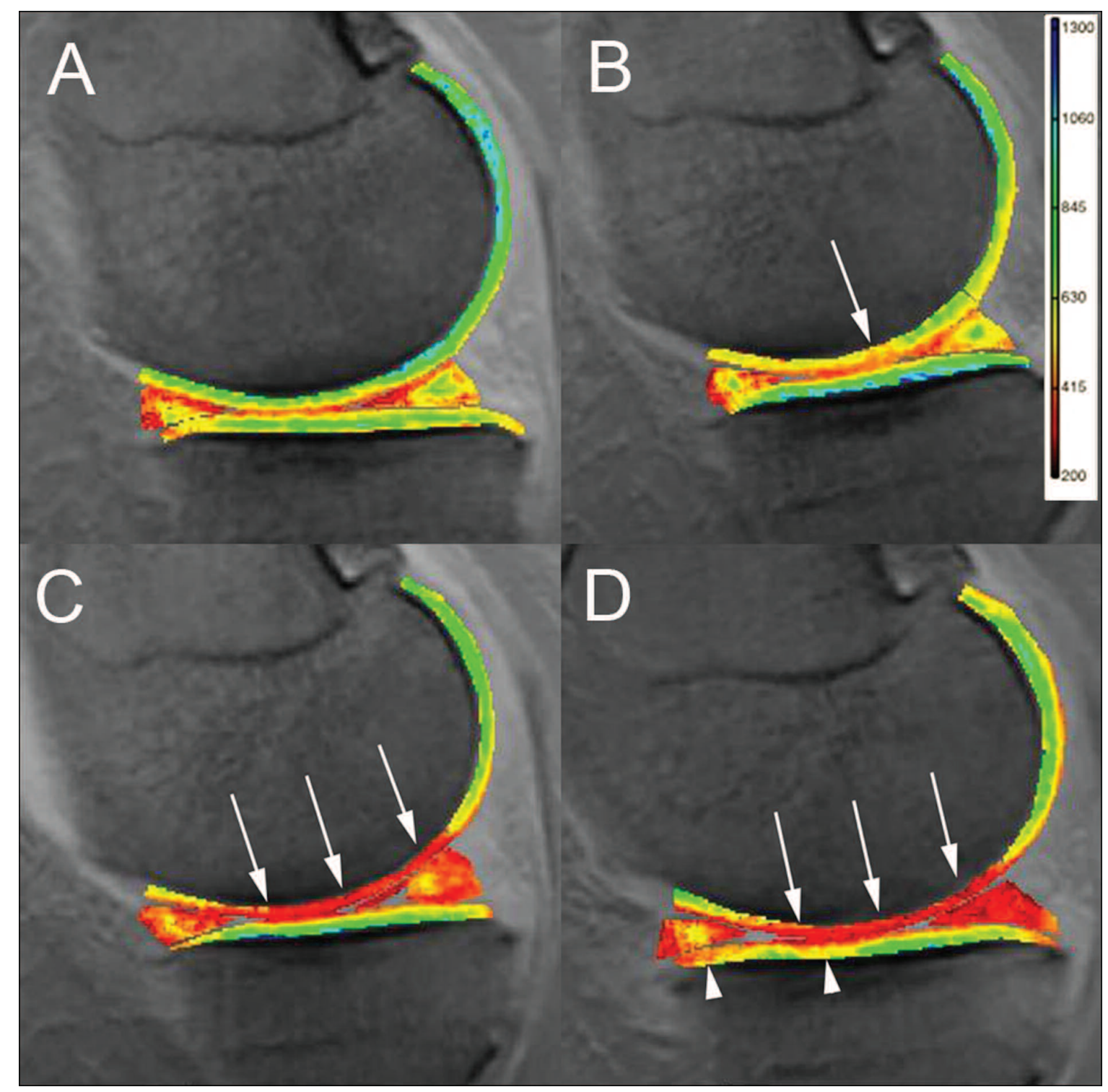

Figure 1. Compositional MRI as a tool to detect longitudinal changes in cartilage matrix over 2 years. Sagittal 3-D inversion recovery-prepared SPRG with selective water excitation images of the medial tibiofemoral compartment acquired 90 min after intravenous injection of gadolinium for dGEMRIC (GAG) assessment at baseline (A), 3 months (B), 1 year (C), and 2 years (D). Baseline assessment (A) shows homogeneous and higher dGEMRIC indices according to the color-coded map within the femoral and tibial cartilage. A progressive decrease in dGEMRIC indices at the central weight-bearing subregion of the medial femur, coded in red, is observed during followup, consistent with a decrease in GAG content (arrows, B, C, and D). Focal areas of decrease in dGEMRIC indices are apparent in the medial tibia at 2 years when compared to previous assessments (arrowheads). Note also a decrease in dGEMRIC indices in the medial meniscus over time. MRI: magnetic resonance imaging; dGEMRIC: delayed gadolinium-enhanced MRI of cartilage; GAG: glycosaminoglycan; SPGR: spoiled gradient echo. Panel C is from Crema, et al. Arthritis Rheumatol 2014;66:1517-24; with permission.

clinical trials. First, it is currently difficult to duplicate the exact sequence after a few years because of changes in equipment, making longterm followup studies difficult. Second, it is difficult to define the threshold of "pathologic value" for indices of cartilage biochemical composition (e.g., T2, dGEMRIC index) because of large inter- and intraindividual variations. Third, although compositional imaging techniques have been explored in other fields such as imaging of cartilage repair and rheumatoid arthritis, there has been no major breakthrough despite availability of these techniques for 2 decades. This may be a reflection of the aforementioned limitations and perhaps due in part to technical difficulties and limited availability of advanced MRI scanners. At least 1 study, however, reported moderate to excellent reproducibility for T1rho and T2 mapping in a multicenter setting. The study included large differences, with intraclass correlation coefficients ranging from 0.61 to 0.98 and root-mean-square coefficients of variation ranging from $4 \%$

Personal non-commercial use only. The Journal of Rheumatology Copyright @ 2016 . All rights reserved. 
to $14 \%$, which seems promising in regard to future multicenter investigations ${ }^{21}$. Newer techniques that are easier to implement such as gagCEST may offer help here.

Compositional MRI techniques seem to have the potential to supplement clinical MRI sequences in identifying cartilage degeneration at an earlier stage than is possible today. Different techniques are complementary, in that some focus on isotropy or the collagen network (e.g., T2 mapping and T1rho) while others focus on tissue composition, e.g., dGEMRIC, that conveys information on the GAG concentration. So far, however, the applicability and responsiveness of these techniques to clinical or structural outcomes have not been well established. In addition to the different tissue components targeted by the different techniques, applicability and feasibility will play an important role in implementation in a larger clinical study, or eventually in clinical practice. While some, such as T2 mapping and dGEMRIC, are easily applied on standard clinical platforms using 1.5 or $3 \mathrm{~T}$ systems, others require dedicated hardware or software. Compositional MRI techniques seem promising in terms of predicting structural and clinical outcomes relevant to OA research, at least for a short-term followup study of up to 1 year. At present, compositional MRI techniques are potentially helpful as adjunct or secondary outcome measures in short-term clinical trials involving OA.

\author{
ALI GUERMAZI, MD, $\mathrm{PhD}$, \\ Professor of Radiology, \\ Director, Quantitative Imaging Center, \\ Boston University School of Medicine, \\ Boston, Massachusetts; \\ MICHEL D. CREMA, MD, \\ Department of Radiology, \\ Boston University School of Medicine, \\ Boston, Massachusetts, USA, and \\ Department of Radiology, \\ Hospital do Coração and Teleimagem, \\ São Paulo, Brazil;
}

FRANK W. ROEMER, MD, Department of Radiology, Boston University School of Medicine, Boston, Massachusetts, USA, and Department of Radiology, University of Erlangen, Erlangen, Germany.

Address correspondence to Dr. A. Guermazi, Boston University School of Medicine, 820 Harrison Ave., FGH Building, 3rd Floor, Boston, Massachusetts 02118, USA; E-mail: guermazi@bu.edu

\section{REFERENCES}

1. Crema MD, Roemer FW, Marra MD, Burstein D, Gold GE, Eckstein F, et al. Articular cartilage in the knee: current MR imaging techniques and applications in clinical practice and research. Radiographics 2011;31:37-61.

2. McAlindon TE, Nuite M, Krishnan N, Ruthazer R, Price LL, Burstein D, et al. Change in knee osteoarthritis cartilage detected by delayed gadolinium enhanced magnetic resonance imaging following treatment with collagen hydrolysate: a pilot randomized controlled trial. Osteoarthritis Cartilage 2011;19:399-405.
3. Baum T, Joseph GB, Arulanandan A, Nardo L, Virayavanich W, Carballido-Gamio J, et al. Association of magnetic resonance imaging-based knee cartilage $\mathrm{T} 2$ measurements and focal knee lesions with knee pain: data from the Osteoarthritis Initiative. Arthritis Care Res 2012;64:248-55.

4. Stehling C, Liebl H, Krug R, Lane NE, Nevitt MC, Lynch J, et al. Patellar cartilage: T2 values and morphologic abnormalities at 3.0-T MR imaging in relation to physical activity in asymptomatic subjects from the osteoarthritis initiative. Radiology 2010;254:509-20.

5. Kijowski R, Blankenbaker DG, Munoz Del Rio A, Baer GS, Graf BK. Evaluation of the articular cartilage of the knee joint: value of adding a T2 mapping sequence to a routine MR imaging protocol. Radiology 2013;267:503-13.

6. Liebl H, Joseph G, Nevitt MC, Singh N, Heilmeier U, Subburaj K, et al. Early T2 changes predict onset of radiographic knee osteoarthritis: data from the osteoarthritis initiative. Ann Rheum Dis 2015;74:1353-9.

7. Krause FG, Klammer G, Benneker LM, Werlen S, Mamisch TC, Weber M. Biochemical T2* MR quantification of ankle arthrosis in pes cavovarus. J Orthop Res 2010;28:1562-68.

8. Kim T, Min BH, Yoon SH, Kim H, Park S, Lee HY, et al. An in vitro comparative study of $\mathrm{T} 2$ and $\mathrm{T} 2 *$ mappings of human articular cartilage at 3-Tesla MRI using histology as the standard of reference. Skeletal Radiol 2014;43:947-54.

9. Stahl R, Luke A, Li X, Carballido-Gamio J, Ma CB, Majumdar S, et al. T1rho, T2 and focal knee cartilage abnormalities in physically active and sedentary healthy subjects versus early OA patients - a 3.0-Tesla MRI study. Eur Radiol 2009;19:132-43.

10. Wang L, Regatte RR. Quantitative mapping of human cartilage at 3.0T: parallel changes in T(2), T(1)rho, and dGEMRIC. Acad Radiol 2014;21:463-71.

11. Owman H, Tiderius CJ, Neuman P, Nyquist F, Dahlberg LE. Association between findings on delayed gadolinium-enhanced magnetic resonance imaging of cartilage and future knee osteoarthritis. Arthritis Rheum 2008;58:1727-30.

12. Crema MD, Hunter DJ, Burstein D, Roemer FW, Krishnan N, Hellio Le Graverand MP, et al. The relationship between the Kellgren-Lawrence grade of radiographic knee osteoarthritis and delayed gadolinium-enhanced MRI of medial tibiofemoral cartilage (dGEMRIC): a 1-year follow-up study. Osteoarthritis Cartilage 2013;21:S179-80.

13. Owman H, Ericsson YB, Englund M, Tiderius CJ, Tjörnstrand J, Roos EM, et al. Association between delayed gadolinium-enhanced MRI of cartilage (dGEMRIC) and joint space narrowing and osteophytes: a cohort study in patients with partial meniscectomy with 11 years of follow-up. Osteoarthritis Cartilage 2014;22:1537-41.

14. Crema MD, Hunter DJ, Burstein D, Roemer FW, Li L, Krishnan N, et al. Delayed gadolinium-enhanced magnetic resonance imaging of medial tibiofemoral cartilage and its relationship with meniscal pathology: a longitudinal study using 3.0T magnetic resonance imaging. Arthritis Rheumatol 2014;66:1517-24.

15. Crema MD, Hunter DJ, Burstein D, Roemer FW, Li L, Eckstein F, et al. Association of changes in delayed gadolinium-enhanced MRI of cartilage (dGEMRIC) with changes in cartilage thickness in the medial tibiofemoral compartment of the knee: a 2 year follow-up study using 3.0 T MRI. Ann Rheum Dis 2014;73:1935-41.

16. van Tiel J, Kotek G, Reijman M, Bos PK, Bron EE, Klein S, et al. Delayed gadolinium-enhanced MRI of the meniscus (dGEMRIM) in patients with knee osteoarthritis: relation with meniscal degeneration on conventional MRI, reproducibility, and correlation with dGEMRIC. Eur Radiol 2014;24:2261-70.

17. Wheaton AJ, Borthakur A, Shapiro EM, Regatte RR, Akella SV, Kneeland JB, et al. Proteoglycan loss in human knee cartilage: quantitation with sodium MR imaging - feasibility study. Radiology 2004;231:900-5. 
18. Madelin G, Babb J, Xia D, Chang G, Krasnokutsky S, Abramson SB, et al. Articular cartilage: evaluation with fluid-suppressed 7.0-T sodium MR imaging in subjects with and subjects without osteoarthritis. Radiology 2013;268:481-91.

19. Raya JG, Horng A, Dietrich O, Krasnokutsky S, Beltran LS, Storey $\mathrm{P}$, et al. Articular cartilage: in vivo diffusion-tensor imaging. Radiology 2012;262:550-9

20. Ling W, Regatte RR, Navon G, Jerschow A. Assessment of glycosaminoglycan concentration in vivo by chemical exchange-dependent saturation transfer (gagCEST). Proc Natl Acad Sci U S A 2008;105:2266-70.

21. Mosher TJ, Zhang Z, Reddy R, Boudhar S, Milestone BN, Morrison WB, et al. Knee articular cartilage damage in osteoarthritis: analysis of MR image biomarker reproducibility in ACRIN-PA 4001 multicenter trial. Radiology 2011;258:832-42.

J Rheumatol 2016;43:7-11; doi:10.3899/jrheum.150663 